\title{
Neutron Experiments at J-PARC
}

\section{Masaaki Kitaguchi*}

Kobayashi-Maskawa Institute for the Origin of Particles and the Universe (KMI), Nagoya

University

E-mail: kitaquchiephi.phys.nagoya-u.ac.jp

The neutron is suitable for precision measurements to search for new physics. Extremely-high intense pulsed neutron provided by J-PARC enable us to perform new type of precision measurements. We are performing various experiments with low energy neutrons at J-PARC.

In this paper, we describe our experimental search for time reversal symmetry violation in nuclear reactions. The enhancement of the T-violation is predicted in the neutron capture reaction for some nuclei. Our recent results of ${ }^{139} \mathrm{La}(\mathrm{n}, \gamma)$ reaction suggested that the enhancement is large enough to search T-violation with high sensitivity, which can be reach to that of neutron EDM and which has different systematics.

In J-PARC, we are also performing the precision measurement of neutron lifetime and mediumrange unknown force search by using pulsed neutrons. We are continuing researches and developments, for example, search for neutron electric dipole moment by using neutron diffraction with non-centrosymmetric crystal and a chameleon field search with neutron interferometer.

The 4th KMI International Symposium (KMI2019)

18-20, February 2019

Nagoya, Japan

${ }^{*}$ Speaker. 


\section{Introduction}

Neutron is a chargeless massive particle with the lifetime in the macroscopic range, which is suitable for precision measurement of the small influence of new physics. The combination of accelerator-driven high-intensity neutron source and high-performance neutron optics enables us to perform various physics experiments with far superior statistics. Now we stand at the portals of a new era of neutron physics.

Japan Proton Accelerator Research Complex (J-PARC) has world-highest intensity pulsed neutron source named as the Japan Spallation Neutron Source (JSNS). Pulsed neutrons are provided with pulse width short enough to achieve good neutron energy resolution and repetition rate low enough to use slow neutrons. Neutron intensity for each pulse is extremely high due to $1 \mathrm{MW}$ of injected proton beam power. These features has the advantage to study fundamental physics with neutrons. The NOP collaboration aims to do the fundamental physics experiments by using neutrons. Various physics experiments are performing, for example, neutron lifetime measurement, medium-range unknown force search, search for neutron electric dipole moment, and so on. In this paper, our status and plan of the search for large time reversal symmetry violation in nuclear reaction is discussed.

\section{T-violation in compound nuclei}

\subsection{Enhancement of T-violation}

Today's matter universe requires $\mathrm{CP}$-violation which is larger than that from standard model of particle physics. The measurements of the neutron static electric dipole moment (nEDM) provided one of the strongest constraints in the search for the large time reversal violation. The present upper limit is $\left|d_{\mathrm{n}}\right|<3 \times 10^{-26} e \cdot \mathrm{cm}$ (90\% C.L.), which is close to predictions for EDMs which arise from new physics [四]. Although next-generation UCN sources are being developed intensively for the sensitivity of the order of $10^{-27} \sim 10^{-28} e \cdot \mathrm{cm}$, it is valuable and important to develop different methods to search the T-violation with the other methods with different systematic uncertainties and hopefully to improve the experimental sensitivity.

In the nuclear interaction studies, the large enhancement of parity $(\mathrm{P})$ violation was discov-

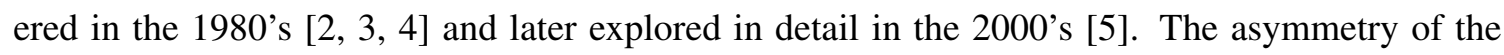
capture cross section with respect to the helicity of incident neutrons was enhanced by at most $10^{6}$ times larger in a several nuclei and at the neutron energy for p-wave resonance peak (fig. $\mathbb{D}$ ). The enhancement is explained as the result of the entrance channel interference between neighboring sand p-wave amplitudes [ $[$,, 0 . The mechanism of the P-violating effect has been proposed theoretically to be applicable to enhance the experimental sensitivity to search the T-violation [8, Q]. The advantage to apply the enhancement mechanism is that takes T-odd effects due to the final-state interaction is expected to be negligibly small as the neutron propagation through the target material can be described by the neutron optics. The cross section to be measured is given as

$$
\Delta \sigma_{\mathrm{T}}=\kappa(J) \frac{W_{\mathrm{T}}}{W} \Delta \sigma_{\mathrm{P}}
$$

where $\Delta \sigma_{\mathrm{T}}$ and $\Delta \sigma_{\mathrm{P}}$ are the T-violating and P-violating cross section, $\kappa(J)$ is the spin factor, $W_{\mathrm{T}}$ and $W$ denote the T- and P-violating matrix elements. This means that the candidate nuclei for 

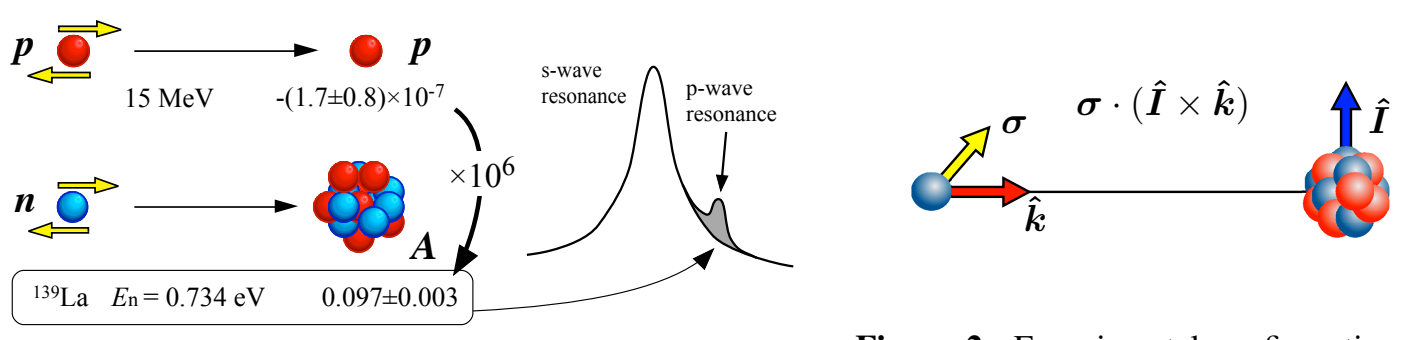

Figure 2: Experimental configuration for T-violation search.

Figure 1: P-violation enhancement around p-wave resonance.

T-violation search are the nuclei with large enhancement of P-violation, however, the parameter $\kappa(J)$ must be measured.

\subsection{Candidates of target nuclei}

Among the nuclei with large $\sigma_{\mathrm{P}}$, preferable nuclei have p-wave resonance peak at the energy of neutron about a few $\mathrm{eV}$ due to the incident neutron energy spectrum of the facility like J-PARC. For example, ${ }^{139} \mathrm{La},{ }^{131} \mathrm{Xe},{ }^{117} \mathrm{Sn},{ }^{81} \mathrm{Br}$, and ${ }^{115} \mathrm{In}$ can be candidates as the target materials. In addition, large natural abundance and small nuclear spin are desirable for actual experiments. ${ }^{139} \mathrm{La}$ is one of the most promising nuclei with the p-wave resonance with the neutron energy of $0.75 \mathrm{eV}$ and with P-violation enhancement of the order of $10^{6}$. The enhancement factor $\kappa(J)$ of ${ }^{139} \mathrm{La}$ was determined experimentally by using ${ }^{139} \mathrm{La}(\mathrm{n}, \gamma)$ measurements.

The differential cross section of the $(\mathrm{n}, \gamma)$ reaction with unpolarized incident neutron and unpolarized nuclear target can be expanded as

$$
\frac{\mathrm{d} \sigma}{\mathrm{d} \Omega}=\frac{1}{2}\left(a_{0}+a_{1} \cos \theta_{\gamma}\right)
$$

for the first order, where $\theta_{\gamma}$ is the direction of the $\gamma$-ray, $a_{0}$ and $a_{1}$ are the coefficients, respectively. According to s-p mixing theory with statistical treatments in the compound nuclei, the coefficients can be described by using the total angular momentum, the spin of incident neutron, the spin of the initial and final states of the nuclei, and the partial neutron width for the incident neutrons to the p-wave resonance [ $[\mathbf{W}]$. On the other hand, the $\kappa$ value can be calculated by using the total angular momentum and the partial neutron width of the p-wave resonance. This means that the $\kappa$ value is determined by extracting the partial neutron width by measuring the angular correlation term $a_{1}$ only with the gated $\gamma$-rays corresponding to the well-known spin-states and to the p-wave resonance.

The measurement was performed at the beamline BL04 ANNRI in J-PARC. The 22 germanium detectors are arranged surrounding the nuclear target to measure the angular distribution of $\gamma$-rays (Fig. [1). According to the energy resolution of the detectors and the intense pulsed neutrons, we can analyze the data for each $\gamma$-ray individually. We successfully observed the angular distribution of the $\gamma$-rays and demonstrated $\kappa(J)$ of the order of $1[\mathbb{W}]$. This lead that the enhancement for T-violation can be $10^{6}$ for ${ }^{130} \mathrm{La}$.

We have also started the development of the polarization of ${ }^{139} \mathrm{La}$ nuclei by using dynamical nuclear polarization (DNP) technique. The lanthanum nuclei in $\mathrm{LaAl}_{2} \mathrm{O}_{3}$ crystal were successfully 


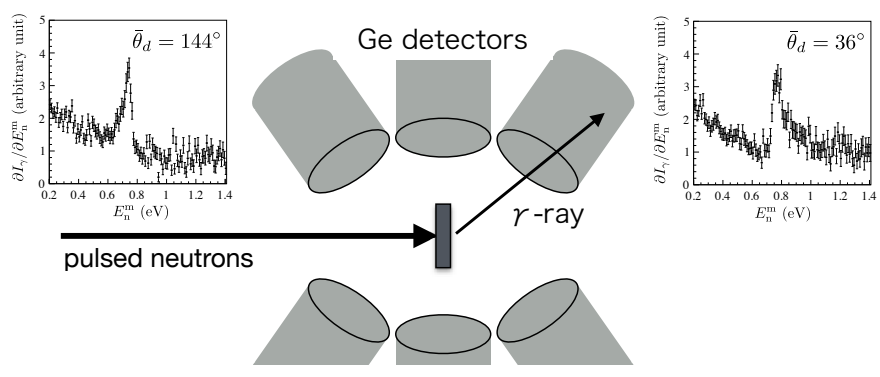

Figure 3: Measurement of the angular distribution of $\gamma$-rays at ANNRI in J-PARC. Clear angular dependence of neutron energy spectrum was observed.

polarized by using DNP method with the polarization of about 50\% [12]. The electrons of paramagnetic impurities $\left(\mathrm{Nd}^{3+}\right)$ in the crystal can be polarized under relatively-high temperature and relatively-low magnetic field. By applying microwave the electron polarization is transferred to the nuclear spin of ${ }^{139} \mathrm{La}$ through a dipole interaction. The perovskite structure of the crystal is suitable to keep the nuclear polarization of ${ }^{139} \mathrm{La}$. Development of large $\mathrm{LaAl}_{2} \mathrm{O}_{3}$ crystal growth and its polarization technique has started with Tohoku University and Osaka University.

The enhancement factor of the other candidate nuclei, ${ }^{131} \mathrm{Xe},{ }^{117} \mathrm{Sn},{ }^{81} \mathrm{Br}$, and ${ }^{115} \mathrm{In}$ are also tried to evaluate by $(\mathrm{n}, \gamma)$ measurements in J-PARC, same as ${ }^{139} \mathrm{La} .{ }^{131} \mathrm{Xe}$ may be polarized by using spin exchange optical pumping (SEOP) technique. In the case of ${ }^{81} \mathrm{Br}$, triplet-DNP method may be applied for its polarization in higher temperature and lower magnetic field. ${ }^{117} \mathrm{Sn}$ has the advantage for the polarization due to its spin of $1 / 2$, although the natural abundance is small.

\subsection{Feasibility of T-violation search at J-PARC}

The neutron-nucleus forward scattering amplitude can be expanded as

$$
f=A+B \boldsymbol{\sigma} \cdot \hat{\boldsymbol{I}}+C \boldsymbol{\sigma} \cdot \hat{\boldsymbol{k}}+D \boldsymbol{\sigma} \cdot(\hat{\boldsymbol{I}} \times \hat{\boldsymbol{k}})
$$

where $\boldsymbol{\sigma}, \hat{\boldsymbol{I}}$ and $\hat{\boldsymbol{k}}$ are the spin of incident neutron, the momentum of the neutron, and the spin of target nuclei, respectively (fig. $\mathbb{Z}$ ). $A$ is the spin independent (P-even, T-even) term. $B$ is the spin dependent (P-even, T-even) term, which is corresponding to neutron spin rotation through the polarized target called as 'pseudomagnetic effect'. $C$ is the P-violating (P-odd, T-even) term, which is enhanced for the case of some nuclei discussed previously. This term can be estimated as the ratio of P-violating cross section to the total capture cross section at the p-wave resonance. $D$ is the T-violating (P-odd, T-odd) term. The measured forward intensity of the beam is proportional to $f^{*} f$, with the value of $A^{*} D$, which is from interference of $A$ and $D$, gives us the largest measurement value as a combination of analyzing power and polarization of transmitted beam.

Each correlation term can be written by using the spin of nuclei, the partial neutron width, and the T-violating weak matrix element. By using the effective field theory (EFT), the matrix element in eq.(…1) is written as

$$
\frac{W_{\mathrm{T}}}{W} \simeq(-0.47)\left(\frac{\bar{g}_{\pi}^{(0)}}{h_{\pi}^{1}}+(0.26) \frac{\bar{g}_{\pi}^{(1)}}{h_{\pi}^{1}}\right),
$$



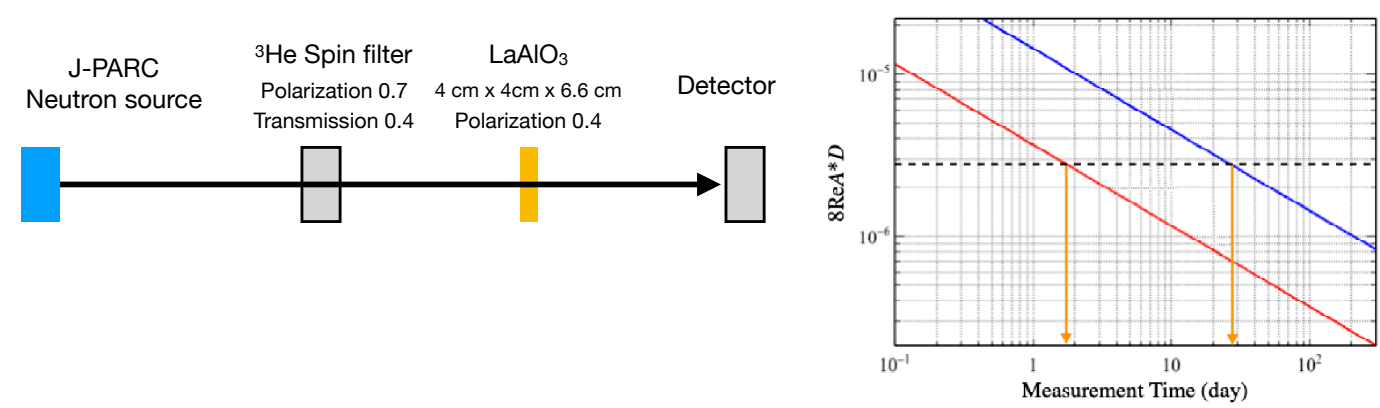

Figure 4: Experimental setup for T-violation search at J-PARC. Right plot shows the sensitivity of asymmetry according to measurement time. Red line indicates ideal case with $100 \%$ of neutron and nuclear polarization. Blue line is actual case with $70 \%$ of neutron polarization and $40 \%$ of target nuclear polarization. Dotted line shows the equivalent of nEDM sensitivity for T-violation.

where $\bar{g}_{\pi}^{(0)}, \bar{g}_{\pi}^{(1)}$ are isoscalar and isovector time reversal invariant meson-nucleon coupling constants, $\bar{h}_{\pi}^{1}$ is a P-violating meson exchange coupling constant. The upper limits of $\bar{g}_{\pi}^{(0)}, \bar{g}_{\pi}^{(1)}$, and The value of $\bar{h}_{\pi}^{1}$ are estimated from the nEDM, ${ }^{199} \mathrm{Hg}$-EDM search, and the measurement of the P-violation of neutron-proton reactions $(\mathrm{n}+\mathrm{p} \rightarrow \mathrm{d}+\gamma)$ respectively as [[13, [4]]

$$
\bar{g}_{\pi}^{(0)}<2.5 \times 10^{-10}, \quad \bar{g}_{\pi}^{(1)}<0.5 \times 10^{-11}, \quad h_{\pi}^{1}=(3.04 \pm 1.23) \times 10^{-7} .
$$

These lead

$$
\left|\frac{W_{\mathrm{T}}}{W}\right|<3.9 \times 10^{-4}
$$

Now the limit of $D$ term can be estimated by using those parameters, $\kappa$ of the order of 1 and $W_{\mathrm{T}} / W$ of the order of $10^{-4}$. We measure experimentally both of asymmetry of capture cross section with polarized incident beam $A_{x}$ and polarization of transmitted beam with unpolarized incident beam $P_{x}$ to extract $8 \operatorname{Re} A^{*} D$. The sensitivity depends on the thickness of the nuclear target because of its transmittance. The optimum thickness is $6.6 \mathrm{~cm}$ of $\mathrm{LaAl}_{2} \mathrm{O}_{3}$ crystal and the maximum experimental sensitivity is $1.1 \times 10^{-5}$ of $8 \operatorname{Re} A^{*} D$. This value means the experimentally discovery potential beyond current upper limit, for example, by nEDM search.

The feasibility for the T-violation search in J-PARC is discussed. We consider a ${ }^{3} \mathrm{He}$ spin filter used to polarize the incident neutrons. ${ }^{3} \mathrm{He}$ spin filter is based on well-established SEOP technique, which can provide the polarization of $70 \%$ of neutrons by using $100 \mathrm{~atm} \cdot \mathrm{cm}{ }^{3} \mathrm{He}$ gas cell. The large size of $\mathrm{LaAl}_{2} \mathrm{O}_{3}$ crystal is required and its polarization of $40 \%$ is assumed. When the beamline at MLF in J-PARC is assumed, the measurement for the asymmetry of the order of $10^{-5}$ can be achieved by using of 30 days (fig. 田).

We must measure the tiny asymmetry of transmitted neutrons in T-violation experiment. Finally neutron detectors which can count the neutron with the rate of the order from $10^{8}$ to $10^{9}$ $\mathrm{cps} / \mathrm{cm}^{2}$ are desirable. Now we have started research and development of the new types of neutron detectors with high counting rate including the electric circuits. 


\section{Neutron Lifetime and search for medium-range unknown force}

The neutron lifetime is an important parameter of the weak interaction and for Big Bang Nucleosynthesis, however, the recent values deviate far beyond the systematic errors claimed in the past. Although some experiments with ultra-cold neutrons provide the results with small uncertainties, the other measurement with cold neutron beam should and with different systematics be performed. We are now measuring the neutron lifetime at BL05 in J-PARC. A polarized neutron beam is chopped into short bunches by a spin flip chopper to avoid the effects from detector walls. A time projection chamber operated with $\mathrm{He}$ and $\mathrm{CO}_{2}$ gas, including a well-controlled amount of ${ }^{3} \mathrm{He}$, is used for detection of the electron from beta decay and determination of the beam intensity. Currently the uncertainty is evaluated as the order of $1 \%$. We are now planning the upgrade in order to increase the incident neutron flux and to study the systematic uncertainties precisely with more statistics.

The inverse-square law of gravity is well-confirmed for the long distance, however, there is room for search for deviations in the case of medium range because of electromagnetic background effects. It can be searched by using neutron scattering on noble gases. The deviation of angular distribution of scattered neutrons from well-known nuclear scattering suggests the unknown force between a noble gas atom and a neutron. This experiment was also performed at BL05 in JPARC. By comparing data of some kind of gases and simulations in order to reduce the systematic uncertainties, we successfully give a new constraint of Yukawa-like unknown interaction in the range of sub-nm [15]. We are continuing the measurements to improve the sensitivity and to measure neutron-electron scattering length precisely.

\section{Other research and developments}

We are planning the nEDM search experiment by measuring spin rotation of polarized neutrons propagating with the diffraction in the noncentrosymmetric crystal. The principle is based on dynamical diffraction theory. In the case of the proper incident angle of neutron wave, the propagation in the crystal can be written as a superposition of two Bloch functions. The interference between the Bloch components results the spin rotation due to EDM induced by the Schwinger effect of the inner electric field. The strong electric field inside the crystal and intense cold-neutron beam can be used for search nEDM. We have observed the clear "Pendellösung fringes", which is one of the proofs of dynamical diffraction by pulsed neutrons at J-PARC. Now we are continuing the research and development.

The spatial distribution of neutron's quantum-mechanical states induced by gravity was observed by using ultra-cold neutrons [1], [8]]. The deviation of the distribution from newton's law can suggest new physics. We have developed ultra-high resolution neutron detector by using nuclear emulsion technique [एव]. Fine-grained nuclear emulsion on ${ }^{10} \mathrm{~B}_{4} \mathrm{C}$ thin layer record the track of particle converted from neutron. The spatial resolution was better than $100 \mathrm{~nm}$.

\section{Acknowledgments}

The neutron scattering experiment was approved by the Neutron Scattering Program Advisory Committee of IMSS, KEK (Proposal No. 2014S03, 2015S12, 2018S12, 2019S03). The neu- 
tron experiment at the Materials and Life Science Experimental Facility of the J-PARC was performed under a user program (Proposal No. 2015A0245, 2015A0316, 2015AU1701, 2016A0227, 2016B0141, 2016B0200, 2016B0202, 2017A0063, 2017A0158, 2017A0170, 2017A0203).

This work was supported by MEXT KAKENHI Grant Number JP19GS0210 and JSPS KAKENHI Grant Number JP16H02194, JP16K13804, JP17H02889. This work was also supported by Toyoaki Foundation.

\section{References}

[1] J. M. Pendlebury et al., Phys. Rev. D 92, 092003 (2015).

[2] M. Forte et al., Phys. Rev. Lett. 45, 2088 (1980).

[3] V. P. Alfimenkov et al., JETP Pis'ma 34, 308 (1981).

[4] V. P. Alfimenkov et al., Nucl. Phys. A398, 93-106 (1983).

[5] G. E. Mitchell, J. D. Bowman, S. I. Pentillä, and E. I. Sharapov, Phys. Rep. 354, 157 (2001).

[6] O. P. Sushkov and V. V. Flambaum, Usp. Fiz. Nauk 136, 3 (1982).

[7] O. P. Sushkov and V. V. Flambaum, Sov. Phys. Uspekhi 25, 1 (1982).

[8] V. P. Gudkov, Phys. Rep. 212, 77 (1992).

[9] J. D. Bowman and V. P. Gudkov, Phys. Rev. C 90, 065503 (2014).

[10] V. V. Flambaum and O. P. Sushkov, Nucl. Phys. A 435, 352-380 (1985).

[11] T. Okudaira et al., Phys. Rev. C 97, 034622 (2018).

[12] T. Maekawa et al., Nucl. Instr. and Meth. A 366, 115-119 (1995).

[13] Y.H.Song, R. Lazauskas, and V. Gudkov. Phys. Rev. C., 83:065503, 2011.

[14] Jason A. Fry. PhD. thesis, 2015.

[15] C. C. Haddock et al., Phys. Rev. D 97, 062002 (2018).

[16] S. Itoh et al., Nucle. Instr. and Meth A908, 78 (2018).

[17] V. V. Nesvizhevsky et al. Nature 415, 297 (2002).

[18] G. Ichikawa et al., Phys. Rev. Lett. 112 (2014) 071101.

[19] N. Naganawa et al., Eur. Phys. J. C 78, 959 (2018). 